\section{Severidad de dehiscencias y fenestraciones en pacientes orto quirúrgicos con maloclusión Clase III evaluados con tomografía computarizada cone beam}

\author{
Severity of dehiscences and fenestrations \\ in ortho-surgical patients with Class III \\ malocclusion evaluated with cone beam \\ computed tomography
}

\begin{abstract}
Resumen
Objetivo. Determinar la frecuencia y severidad de las dehiscencias y fenestraciones vestibulares en las piezas anteriores de pacientes orto quirúrgicos con maloclusión de Angle Clase III evaluados con tomografía computarizada de haz cónico pre quirúrgica o tomografía computarizada cone beam. Métodos. Se evaluaron 30 tomografías de pacientes con maloclusión Clase III esquelética con tratamiento de ortodoncia pre quirúrgica. La muestra fue no probabilística, de casos consecutivos, atendidos en la Facultad de Odontología de la Universidad Nacional Mayor de San Marcos y en el Servicio de Odontología del Hospital Nacional Guillermo Almenara Irigoyen durante el año 2018. Se consideró dehiscencia a la migración apical de la cresta alveolar a partir de $2 \mathrm{~mm}$ desde la unión cemento-esmalte y fenestración, a la porción radicular expuesta sin afectar el margen óseo a partir de $0,5 \mathrm{~mm}$. Resultados. Del total de tomografías, $43,3 \%$ fueron de mujeres y $56,7 \%$ fueron de varones. Se observaron dehiscencias en todas las tomografías, con mayor frecuencia en la mandíbula $(91,7 \%)$ y caninos inferiores (100\%). Las fenestraciones se observaron en el $66,7 \%$, con mayor frecuencia en el maxilar $(28,3 \%)$ y caninos superiores $(31,7 \%)$. El grado severo fue más frecuente en dehiscencias $(65,8 \%)$ y fenestraciones $(13,9 \%)$, afectando los caninos inferiores $(100 \%)$ y superiores $(26,7 \%)$, respectivamente en cada defecto. Conclusiones. Las dehiscencias estuvieron presentes en todas las tomografías, afectando a los caninos inferiores en el grado severo y las fenestraciones estuvieron presentes en la mayoría de las tomografías, afectando con mayor frecuencia a los caninos superiores en el grado severo.
\end{abstract}

Palabras clave: Maloclusión de Angle Clase III; Tomografía Computarizada de Haz Cónico; Diente canino (fuente: DeCS BIREME).

\begin{abstract}
Objective. Determine the frequency and severity of vestibular dehiscences and fenestrations of anterior teeth in orthodontic-surgical patients with skeletal malocclusion, Angle's Class III; evaluated with presurgical cone-beam computed tomography Methods. Thirty cone-beam computed tomographies of skeletal malocclusion Class III patients
\end{abstract}

ISSN-L 1560-9111; eISSN: 1609-8617

Artículo Original

Carol del Pilar Vásquez Cabrejos 1,a, Percy Romero Tapia 2,b, Gianmarco Rivas Romero 1,a, Gabriela Sedano Balbin 1,a

${ }^{1}$ Universidad Nacional Mayor de San Marcos, Facultad de Odontología. Lima, Perú.

${ }^{2}$ Hospital Nacional Guillermo Almenara Irigoyen, Servicio de Odontología, Lima, Perú.

a Cirujano Dentista.

${ }^{\mathrm{b}}$ Magíster en Estomatología.

\section{Correspondencia:}

Carol del Pilar Vásquez Cabrejos:

pvasquezc2803@gmail.com

Calle Vargas Prada 105 dpto 402. Las Torres de Lima-

tambo. San Borja. Lima, Perú.

ORCID: 0000-0002-8251-0711

\section{Coautores: \\ Percy Romero Tapia \\ drpercyromero@hotmail.com \\ Gianmarco Rivas Romero \\ gian210892@gmail.com \\ Gabriela Sedano Balbin \\ gaby.sedano.balbin@gmail.com}

Editora:

Rosse Mary Falcón-Antenucci

Universidad Inca Garcilaso de la Vega, Perú.

Conflicto de intereses: los autores declaran no tener conflictos de interés.

Fuente de financiamiento: autofinanciado.

Recibido: 28/07/19

Aceptado: $16 / 10 / 19$

Publicado: 24/02/20 
with presurgical orthodontic treatment; were evaluated. The sample consisted of on probabilistic and consecutive cases seen at the Universidad Nacional Mayor de San Marcos Dental School and at the Guillermo Almenara Irigoyen National Hospital's Dental Service in Lima, Perú in 2018. The dehiscence was considered as the apical migration of the alveolar margin bone starting at $2 \mathrm{~mm}$ from the cementoenamel junction, and the fenestration was considered as the exposure of the root portion, excluding the alveolar margin bone; starting at $0.5 \mathrm{~mm}$. Results. Out of all tomographies, $43.3 \%$ were from women and $56.7 \%$ were from men. Dehiscences were observed in all tomographies, most frequently in the mandible (91.6\%) and inferior canines (100\%). Fenestrations were observed in $66.7 \%$, most frequently in the maxilla $(28.3 \%)$ and maxillary canines $(31.7 \%)$. The severe level was more frequently in dehiscences (65.8\%) and fenestrations (13.9\%), affecting the inferior canines (100\%) and maxillary canines (26.7\%), respectively in each defect. Conclusions. Dehiscences were observed in all tomographies, affecting most frequently mandible canines in severe level and fenestrations were observed in most tomographies, affecting most frequently maxillary canines in severe level.

Keywords: Mandibular condyle; Cone-beam computed tomography; Orthognathic surgical procedures; Prognathism (source: MeSH NLM).

\section{Introducción}

La presencia de defectos óseos alveolares y su relación con el tratamiento ortodóntico ha sido tema de discusión desde hace mucho tiempo ${ }^{1,2}$. Los incisivos superiores protruídos y los incisivos inferiores retraídos, debido a la compensación fisiológica en pacientes con maloclusión Clase III, en conjunto con la anatomía ósea reducida que rodean estas piezas dentarias (sínfisis mandibular y placas vestibular, lingual/palatina) restringirán la cantidad de desplazamiento que se pueda realizar durante el tratamiento, especialmente si las inclinaciones requeridas, son excesivas ${ }^{3}$.

Kook et al. ${ }^{4}$ mostraron que hay una mayor pérdida ósea alveolar vertical en los incisivos inferiores, en los pacientes con maloclusión Clase III y que el espesor óseo alveolar en la unión cemento-esmalte (UCE) es menor que en la zona apical, determinando una mayor prevalencia de dehiscencias óseas en estos pacientes, haciéndolos más vulnerables durante el tratamiento de ortodoncia. Además, Sun et al. ${ }^{5}$ encontraron una mayor frecuencia de dehiscencias que de fenestraciones; donde la pieza dentaria más afectada por dehiscencia fue el canino inferior y la más afectada por fenestración fue el canino superior. Incluso, corroboró la exactitud de la tomografía computarizada cone beam (TCCB) para la detección de estos defectos alveolares ${ }^{6,7}$.

Caballero ${ }^{2}$ indicó que los pacientes sin tratamiento de ortodoncia presentaban dehiscencias en piezas dentarias anteriores (incisivos inferiores) y fenestraciones en piezas dentarias posteriores (premolares superiores). Mientras que, los pacientes con tratamiento de ortodoncia, podían presentar una mayor cantidad de dehiscencias y fenestraciones ocasionadas o agravadas por los movimientos ortodónticos, especialmente por el movimiento labio-lingual, disminuyendo la cresta y el espesor óseo alveolar en dirección al movimiento dentario. Durante el tratamiento de ortodoncia se pueden producir defectos alveolares, si las fuerzas que se aplican son mayores a las que puede soportar el periodonto, considerando las características óseas y dentarias que presente el paciente.
En ocasiones, cuando el tratamiento de camuflaje realizado no tiene los resultados esperados, se puede optar por cambiar el tratamiento y realizar la corrección quirúrgica; sin embargo, esto puede perjudicar aún más al paciente ${ }^{8}$. Durante el tratamiento ortodóntico, un factor de riesgo periodontal a considerar es el adelgazamiento cortical causado por los movimientos dentarios. Las paredes corticales labiales y linguales, así como, la sínfisis mandibular son consideradas barreras para la compensación dentoalveolar, por ello, se enfatiza la necesidad de un análisis meticuloso de los tejidos óseos circundantes para la toma adecuada de decisiones ${ }^{9}$.

La presente investigación permite aumentar el conocimiento sobre los defectos óseos, conocidos como dehiscencias y fenestraciones, los cuales pueden generar consecuencias en el tratamiento y un pronóstico desfavorable, debido al daño irreparable del soporte óseo. Es importante relacionar la masa dentaria con la masa esquelética, conociendo cuáles son los límites de trabajo que se tienen disponibles, permitiendo que el diente se encuentre en una posición lo más equidistante posible a las tablas óseas, evitando la formación de dehiscencias y fenestraciones en el hueso alveolar. De esta manera, el propósito de este estudio fue determinar la frecuencia y severidad de las dehiscencias y fenestraciones vestibulares, en las piezas dentarias anteriores de pacientes orto quirúrgicos con maloclusión Clase III evaluados por medio de una TCCB pre quirúrgica.

\section{Métodos}

La investigación es de tipo observacional, transversal, descriptivo y retrospectivo. Muestra no probabilística, por casos consecutivos de 30 pacientes previamente diagnosticados, con confirmación imagenológica, de maloclusión Clase III esquelética que se encontraban con tratamiento de ortodoncia pre quirúrgica.

Las tomografías evaluadas fueron parte de los exámenes auxiliares necesarios en los pacientes con maloclusión Clase III con tratamiento de ortodoncia pre quirúrgica, debido a que se utilizó el mismo formato del consenti- 
miento informado para el tratamiento especializado de estos pacientes, solicitados por la clínica de posgrado de la Facultad de Odontología de la Universidad Nacional Mayor de San Marcos y del Servicio de Odontología del Hospital Nacional Guillermo Almenara Irigoyen.

El presente trabajo de investigación fue un aprovechamiento de los datos ya establecidos en los registros tomográficos donde se obtuvieron los permisos correspondientes para acceder a estos datos.

Análisis tomográfico. Las tomografías se obtuvieron mediante el equipo Point 3D Combi 500 (PointNix Corea), con una exposición de $90 \mathrm{kV}, 5 \mathrm{~mA} \mathrm{y} \mathrm{19s.} \mathrm{El}$ grosor de corte de las imágenes para evaluación fue de 0 $\mathrm{mm}$, el tamaño de vóxel fue de $0,303 \mathrm{~mm}$ y $0,4 \mathrm{~mm}$. La unidad dispone de cuatro campos de visión (FOV) denominados axial, sagital, coronal y $3 \mathrm{D}$ que se utilizaron para ubicar el defecto en el eje mayor de la pieza dentaria. El tamaño de ventana es de 19,5 (ancho) x 24,4 cm (altura), con una resolución de 3,94 lp/mm, el tiempo máximo de escaneo de 20 segundos. Las imágenes fueron analizadas en el software Real Scan 2.0.

Se evaluaron 12 piezas dentarias anteriores por tomografía para realizar las mediciones de dehiscencias y fenestraciones ( 6 superiores y 6 inferiores).

Puntos de referencia y medidas. Los puntos de referencia y medidas se tomaron en la ventana sagital de la TCCB siguiendo el eje mayor de la pieza dentaria ${ }^{5,10,11}$.

Las referencias para tomar las medidas de los defectos (Figura) se obtuvieron de los estudios de Sun ${ }^{5}$ y Lee ${ }^{10}$, considerando, solo el lado vestibular de las piezas dentarias. Estas referencias se describen a continuación.

Dehiscencias: migración apical de la cresta alveolar ${ }^{12}$.

Punto A: unión cemento - esmalte (UCE) ubicada en la superficie de la cara vestibular.
Punto B: cresta alveolar (CA) definida como, el nivel más coronal del hueso alveolar, ubicada en la superficie de la cara vestibular.

La unión de ambos puntos se denominó dehiscencia (d) cuando la medida fue mayor a $2 \mathrm{~mm}$.

Fenestraciones: porción radicular expuesta sin afectar el margen óseo ${ }^{13}$.

Punto C: borde más coronal de la pérdida ósea, en la cara vestibular ubicada en el tercio medio y apical de la pieza dentaria, sin afectar el margen alveolar.

Punto D: borde más apical de la pérdida ósea, en la cara vestibular ubicada en el tercio medio y apical de la pieza dentaria, sin afectar el margen alveolar.

La unión de ambos puntos se denominó fenestración (f) cuando la medida fue mayor a $0,5 \mathrm{~mm}$.

Clasificación del grado de severidad. La severidad se clasificó de la siguiente manera.

Dehiscencias: el rango de clasificación se llevó a cabo tomando como referencia la profundidad de sondaje, establecida por la Academia Americana de Periodoncia (AAP) ${ }^{14}$, debido a que en ambos casos la zona a evaluar manifiesta una migración hacia apical de la cresta ósea, donde la Clase 1 (ausente) se considera hasta los $3 \mathrm{~mm}$, la Clase 2 (leve) hasta los $5 \mathrm{~mm}$, la Clase 3 (moderado) hasta los $7 \mathrm{~mm}$ y la Clase 4 (severo) mayor a $7 \mathrm{~mm}$. Entonces, según los estudios de Sun et al. ${ }^{5}$ y Lee et al. ${ }^{8}$ la dehiscencia se tomó a partir de $2 \mathrm{~mm}$, por ello el grado leve se consideró de $2 \mathrm{~mm}$ a $5 \mathrm{~mm}$, el grado moderado hasta los $7 \mathrm{~mm}$ y el grado severo cuando el defecto es mayor a $7 \mathrm{~mm}$ (Tablas 1 y 2 ).

Fenestraciones: el rango de clasificación se llevó a cabo según la pérdida de inserción clínica, establecida por la AAP ${ }^{14}$, debido a que ambos manifiestan una
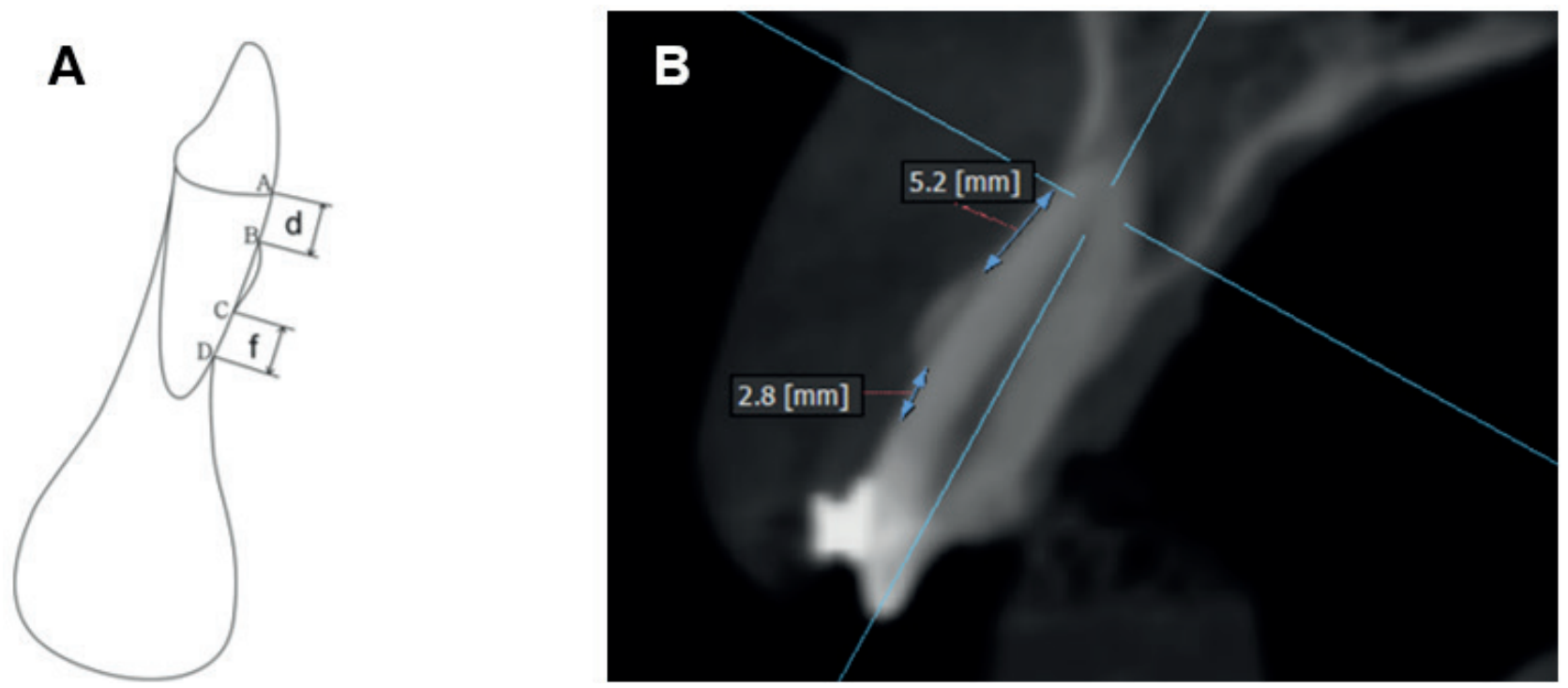

Figura. Ubicación de los puntos críticos para medir las dehiscencias y fenestraciones en una pieza dentaria anterior. A. Modificación de la figura siguiendo el modelo de Sun et al. ${ }^{5}$ Donde la unión del punto A (unión cemento - esmalte) al punto B (cresta alveolar) es considerada una dehiscencia y la unión del punto $C$ (borde más coronal del defecto) al punto $D$ (borde más apical del defecto) es considerada una fenestración en tanto no afecte el margen óseo. B. Ubicación de los puntos A, B, C y D en la vista sagital de la TCCB. 
pérdida ósea, donde la Clase 1 (ausente) se considera en $0 \mathrm{~mm}$, la Clase 2 (leve) hasta los $2 \mathrm{~mm}$, la Clase 3 (moderado) hasta los $4 \mathrm{~mm}$ y la Clase 4 (severo) mayor a $4 \mathrm{~mm}$. Entonces, según el estudio de Hilario ${ }^{15}$ la fenestración se tomó a partir de $0,5 \mathrm{~mm}$ para evitar cualquier error tomográfico, por ello el grado leve se consideró de $0,5 \mathrm{~mm}$ a $2 \mathrm{~mm}$, el grado moderado hasta los $4 \mathrm{~mm}$ y el grado severo cuando el defecto es mayor a $4 \mathrm{~mm}$ (Tablas 1 y 2 ).

Análisis estadístico. El análisis y procesamiento de datos se realizaron a través del programa estadístico SPSS (Versión 23.0 para Windows, IBM, EE.UU.). Los datos se evaluaron mediante estadística descriptiva, con tablas de frecuencia para determinar la presencia de dehiscencias y fenestraciones. Los datos fueron cualificados por grado de severidad según la Tabla 2, realizando el análisis por tipo de pieza dentaria y ubicación (maxilar y mandíbula). Además, se realizó la prueba estadística de Chi-cuadrado para determinar la significancia estadística de los datos obtenidos $(\mathrm{p}<0,05)$.

\section{Resultados}

Descripción de la muestra. De las 30 TCCB de pacientes atendidos con diagnóstico de maloclusión Clase III esquelética, con tratamiento de ortodoncia pre quirúrgica: 13 fueron mujeres y 17 fueron varones, que representaron el $43,3 \%$ y $56,7 \%$ respectivamente.
Frecuencia de dehiscencias y fenestraciones. De las 12 piezas dentarias evaluadas por tomografía, el 100\% de las tomografías presentaron entre 6 y 12 piezas dentarias afectadas por dehiscencias como mínimo y máximo, respectivamente (Tabla 3).

Se encontró al menos una pieza dentaria afectada por fenestración en el 66,7\% de las tomografías evaluadas, a diferencia del 33,3\% de tomografías, que no presentaron ninguna pieza dentaria afectada. Además, el número máximo de fenestraciones por tomografía fue de 9 , en el $10 \%$ de las tomografías (Tabla 4).

Frecuencia de dehiscencias y fenestraciones según ubicación de la arcada. Las dehiscencias se encontraron tanto en maxilar como en mandíbula en todas las tomografías evaluadas. Al evaluar la cantidad de piezas dentarias afectadas por arcada, se pudo observar que la mandíbula fue la más afectada con $91,7 \%$, a diferencia del $8,3 \%$, que no fue afectado (Tabla 5).

El 60\% de las tomografías evaluadas presentaron piezas dentarias afectadas por fenestración en el maxilar, frente al $36,7 \%$, que presentaron piezas dentarias afectadas en mandíbula. Al evaluar la cantidad de piezas dentarias afectadas se obtuvo que, el $28,3 \%$ de fenestraciones se ubicaron en el maxilar y el $16,7 \%$, en la mandíbula (Tabla 6).

Tabla 1. Clasificación del grado de severidad periodontal según la Academia Americana de Periodoncia (AAP) ${ }^{14}$

\begin{tabular}{|c|c|c|c|c|}
\hline \multicolumn{2}{|c|}{ Grado clínico de gravedad } & \multirow{2}{*}{$\begin{array}{c}\text { Inflamación gingival, } \\
\text { hemorragia (BOP) }\end{array}$} & \multirow{2}{*}{$\begin{array}{l}\text { Profundidad de sondaje } \\
\text { (PS) }\end{array}$} & \multirow{2}{*}{$\begin{array}{l}\text { Pérdida de inserción clínica } \\
\text { (PI) }\end{array}$} \\
\hline Clase & Forma & & & \\
\hline Clase 1 & Gingivitis & + hasta +++ & $1-3 \mathrm{~mm}$ & - \\
\hline Clase 2 & Periodontitis leve & + hasta +++ & $4-5 \mathrm{~mm}$ & $1-2 \mathrm{~mm}$ \\
\hline Clase 3 & Periodontitis moderada & + hasta +++ & $6-7 \mathrm{~mm}$ & $3-4 \mathrm{~mm}$ \\
\hline Clase 4 & Periodontitis grave & + hasta +1+ & $>7 \mathrm{~mm}$ & $\geq 5 \mathrm{~mm}$. \\
\hline
\end{tabular}

Tabla 2. Grado de severidad de las dehiscencias y fenestraciones propuesto por el presente estudio tomando como referencia los datos de la AAP

\begin{tabular}{ccc}
\hline & Dehiscencia & Fenestración \\
\hline Ausente & $<2 \mathrm{~mm}$ & $<0,5 \mathrm{~mm}$ \\
Leve & $\geq 2$ hasta $5 \mathrm{~mm}$ & $\geq 0,5$ hasta $2 \mathrm{~mm}$ \\
Moderado & $>5$ hasta $7 \mathrm{~mm}$ & $>2$ hasta $4 \mathrm{~mm}$ \\
Severo & $>7 \mathrm{~mm}$ & $>4 \mathrm{~mm}$ \\
\hline
\end{tabular}

Tabla 3. Frecuencia de dehiscencias por tomografía

\begin{tabular}{cccc}
\hline \multirow{2}{*}{$\begin{array}{c}\text { Número de piezas dentarias } \\
\text { con dehiscencias }\end{array}$} & $\mathbf{n}$ & TCCB & \\
\cline { 2 - 4 } & 12 & 40 & \% Acum. \\
\hline 12 & 3 & 10 & 40 \\
11 & 6 & 20 & 50 \\
10 & 4 & 13,3 & 70 \\
9 & 2 & 6,7 & 83,3 \\
8 & 1 & 3,3 & 90 \\
7 & 2 & 6,7 & 93,3 \\
6 & 30 & 100 & 100 \\
\hline Total & & & 100 \\
\hline
\end{tabular}


Frecuencia de dehiscencias y fenestraciones según tipo de pieza dentaria anterior. Las dehiscencias fueron encontradas en todos los caninos inferiores evaluados (100\%). La segunda pieza dentaria más afectada fue el incisivo lateral inferior $(88,3 \%)$ y las menos afectadas fueron el incisivo central superior $(76,7 \%)$ y el canino superior $(76,7 \%)$ (Tabla 7$)$.

El 31,7\% de los caninos superiores fueron los más afectados por fenestraciones, seguido del incisivo lateral superior (30\%) y el incisivo central inferior (30\%). A diferencia del canino inferior, que no fue afectado por este defecto en ningún caso (Tabla 8).

Grado de severidad de las dehiscencias. Se observó que, del total de piezas dentarias evaluadas, el 65,8\% fueron afectadas en grado severo, seguido del 17,5\%, que fue afectado en grado leve y del 2,2\%, que fue afectado en grado moderado. Además, en el grado leve, la pieza dentaria más afectada fue el incisivo lateral superior (35\%); en el grado moderado, el incisivo lateral inferior $(6,7 \%)$ y en el grado severo, el canino inferior (100\%) (Tabla 9).

Grado de severidad de las fenestraciones. Se observó que, del total de piezas dentarias evaluadas, el 13,9\% fueron afectadas en grado severo, seguido del 5\%, que fue afectado en grado moderado y del 3,6\%, que fue afectado en grado leve. A diferencia del, 77,5\% de las piezas dentarias evaluadas, que no estuvieron afectadas. Además, en el grado leve, la pieza dentaria más afectada fue el incisivo central inferior $(11,7 \%)$, de igual manera, en el grado moderado (10\%) y en el grado severo, la pieza dentaria más afectada fue el canino superior $(26,7 \%)$ (Tabla 10).

\section{Discusión}

El presente estudio permite reconocer, medir y clasificar mediante la evaluación tomográfica las dehiscencias y fenestraciones en pacientes con maloclusión Clase III con tratamiento de ortodoncia pre quirúrgica. Estudios

Tabla 4. Frecuencia de fenestraciones por tomografía

\begin{tabular}{cccc}
\hline \multirow{2}{*}{$\begin{array}{c}\text { Número de piezas dentarias } \\
\text { con fenestraciones }\end{array}$} & $\mathbf{n}$ & TCCB \\
\cline { 2 - 4 } & $\mathbf{n}$ & 10 & \% Acum. \\
\hline 9 & 1 & 3,3 & 10 \\
7 & 1 & 3,3 & 13,3 \\
6 & 2 & 6,7 & 23,3 \\
5 & 2 & 6,7 & 30 \\
4 & 4 & 13,3 & 43,3 \\
3 & 4 & 13,3 & 56,7 \\
2 & 3 & 10 & 66,7 \\
1 & 10 & 33,3 & 100 \\
0 & 30 & 100 & 100 \\
\hline Total & & & \\
\hline
\end{tabular}

Tabla 5. Frecuencia de dehiscencias según ubicación de la arcada

\begin{tabular}{|c|c|c|c|c|c|c|c|c|c|c|c|c|}
\hline & \multicolumn{4}{|c|}{ Maxilar } & \multicolumn{4}{|c|}{ Mandíbula } & \multicolumn{4}{|c|}{ Total } \\
\hline & \multicolumn{2}{|c|}{ ТССВ } & \multicolumn{2}{|c|}{ Dientes } & \multicolumn{2}{|c|}{ ТССВ } & \multicolumn{2}{|c|}{ Dientes } & \multicolumn{2}{|c|}{ ТССВ } & \multicolumn{2}{|c|}{ Dientes } \\
\hline & $\mathbf{n}$ & $\%$ & $\mathbf{n}$ & $\%$ & $\mathbf{n}$ & $\%$ & $\mathbf{n}$ & $\%$ & $\mathbf{n}$ & $\%$ & $\mathbf{n}$ & $\%$ \\
\hline Presenta & 30 & 100 & 143 & 79,4 & 30 & 100 & 165 & 91,7 & 30 & 100 & 308 & 85,6 \\
\hline No Presenta & 0 & 0 & 37 & 20,6 & 0 & 0 & 15 & 8,3 & 0 & 0 & 52 & 14,4 \\
\hline Total & 30 & 100 & 180 & 100 & 30 & 100 & 180 & 100 & 30 & 100 & 360 & 100 \\
\hline
\end{tabular}

(p: 0,001)

Tabla 6. Frecuencia de fenestraciones según ubicación de la arcada

\begin{tabular}{|c|c|c|c|c|c|c|c|c|c|c|c|c|}
\hline & \multicolumn{4}{|c|}{ Maxilar } & \multicolumn{4}{|c|}{ Mandíbula } & \multicolumn{4}{|c|}{ Total } \\
\hline & \multicolumn{2}{|c|}{ TCCB } & \multicolumn{2}{|c|}{ Dientes } & \multicolumn{2}{|c|}{ TCCB } & \multicolumn{2}{|c|}{ Dientes } & \multicolumn{2}{|c|}{ TCCB } & \multicolumn{2}{|c|}{ Dientes } \\
\hline & $\mathbf{n}$ & $\%$ & $\mathbf{n}$ & $\%$ & $\mathbf{n}$ & $\%$ & $\mathbf{n}$ & $\%$ & n & $\%$ & $\mathbf{n}$ & $\%$ \\
\hline Presenta & 18 & 60 & 51 & 28,3 & 11 & 36,7 & 30 & 16,7 & 20 & 66,7 & 81 & 22,5 \\
\hline No Presenta & 12 & 40 & 129 & 71,7 & 19 & 63,3 & 150 & 83,3 & 10 & 33,3 & 279 & 77,5 \\
\hline Total & 30 & 100 & 180 & 100 & 30 & 100 & 180 & 100 & 30 & 100 & 360 & 100 \\
\hline
\end{tabular}

(p: 0,008) 
Tabla 7. Frecuencia de dehiscencias según tipo de pieza dentaria anterior

\begin{tabular}{|c|c|c|c|c|c|c|}
\hline \multirow{3}{*}{ Tipo de pieza dentaria } & \multicolumn{6}{|c|}{ Dehiscencias } \\
\hline & \multicolumn{2}{|c|}{ Presenta } & \multicolumn{2}{|c|}{ No presenta } & \multicolumn{2}{|c|}{ Total } \\
\hline & $\mathbf{n}$ & $\%$ & $\mathrm{n}$ & $\%$ & $\mathbf{n}$ & $\%$ \\
\hline $\mathrm{CI}$ & 60 & 100 & 0 & 0 & 60 & 100 \\
\hline ILI & 53 & 88,3 & 7 & 11,7 & 60 & 100 \\
\hline $\mathrm{ICI}$ & 52 & 86,7 & 8 & 13,3 & 60 & 100 \\
\hline ILS & 51 & 85 & 9 & 15 & 60 & 100 \\
\hline ICS & 46 & 76,7 & 14 & 23,3 & 60 & 100 \\
\hline CS & 46 & 76,7 & 14 & 23,3 & 60 & 100 \\
\hline Total & 308 & 85,6 & 52 & 14,4 & 360 & 100 \\
\hline
\end{tabular}

(p: 0,003)
Cl: Canino Inferior
ILS: Incisivo Lateral Superior
ILI: Incisivo Lateral Inferior
ICS: Incisivo Central Superior
ICI: Incisivo Central Inferior
CS: Canino Superior

Tabla 8. Frecuencia de fenestraciones según tipo de pieza dentaria anterior

\begin{tabular}{|c|c|c|c|c|c|c|}
\hline \multirow{3}{*}{ Tipo de pieza dentaria } & \multicolumn{6}{|c|}{ Fenestraciones } \\
\hline & \multicolumn{2}{|c|}{ Presenta } & \multicolumn{2}{|c|}{ No presenta } & \multicolumn{2}{|c|}{ Total } \\
\hline & $\mathbf{n}$ & $\%$ & $\mathbf{n}$ & $\%$ & $\mathbf{n}$ & $\%$ \\
\hline CS & 60 & 31,7 & 41 & 68,3 & 60 & 100 \\
\hline ILS & 18 & 30 & 42 & 70 & 60 & 100 \\
\hline ICI & 18 & 30 & 42 & 70 & 60 & 100 \\
\hline ICS & 14 & 23,3 & 46 & 76,7 & 60 & 100 \\
\hline ILI & 12 & 20 & 48 & 80 & 60 & 100 \\
\hline $\mathrm{CI}$ & 0 & 0 & 60 & 100 & 60 & 100 \\
\hline Total & 81 & 22,5 & 279 & 77,5 & 360 & 100 \\
\hline
\end{tabular}

$(p<0,001)$

CS: Canino Superior

ILS: Incisivo Lateral Superior

ICI: Incisivo Central Inferior
ICS: Incisivo Central Superior

ILI: Incisivo Lateral Inferior

Cl: Canino Inferior

Tabla 9. Grado de severidad de las dehiscencias según tipo de pieza dentaria anterior

\begin{tabular}{|c|c|c|c|c|c|c|c|c|c|c|}
\hline \multirow{2}{*}{$\begin{array}{c}\text { Pieza } \\
\text { Dentaria }\end{array}$} & \multicolumn{2}{|c|}{ Ausente } & \multicolumn{2}{|c|}{ Leve } & \multicolumn{2}{|c|}{ Moderado } & \multicolumn{2}{|c|}{ Severo } & \multicolumn{2}{|c|}{ Total } \\
\hline & $\mathbf{n}$ & $\%$ & $\mathbf{n}$ & $\%$ & $\mathbf{n}$ & $\%$ & $\mathbf{n}$ & $\%$ & $\mathbf{n}$ & $\%$ \\
\hline ICS & 14 & 23,3 & 17 & 28,3 & 2 & 3,3 & 27 & 45 & 60 & 100 \\
\hline ILS & 9 & 15 & 21 & 35 & 1 & 1,7 & 29 & 48,3 & 60 & 100 \\
\hline CS & 14 & 23,3 & 8 & 13,3 & 1 & 1,7 & 37 & 61,7 & 60 & 100 \\
\hline ICI & 8 & 13,3 & 11 & 18,3 & 0 & 0 & 41 & 68,3 & 60 & 100 \\
\hline ILI & 7 & 11,7 & 6 & 10 & 4 & 6,7 & 43 & 71,7 & 60 & 100 \\
\hline $\mathrm{CI}$ & 0 & 0 & 0 & 0 & 0 & 0 & 60 & 100 & 60 & 100 \\
\hline Total & 52 & 14,4 & 63 & 17,5 & 8 & 2,2 & 237 & 65,8 & 60 & 100 \\
\hline
\end{tabular}


Tabla 10. Grado de severidad de las fenestraciones según tipo de pieza dentaria anterior

\begin{tabular}{|c|c|c|c|c|c|c|c|c|c|c|}
\hline \multirow{2}{*}{$\begin{array}{c}\text { Pieza } \\
\text { Dentaria }\end{array}$} & \multicolumn{2}{|c|}{ Ausente } & \multicolumn{2}{|c|}{ Leve } & \multicolumn{2}{|c|}{ Moderado } & \multicolumn{2}{|c|}{ Severo } & \multicolumn{2}{|c|}{ Total } \\
\hline & $\mathbf{n}$ & $\%$ & $\mathbf{n}$ & $\%$ & $\mathbf{n}$ & $\%$ & $\mathrm{n}$ & $\%$ & $\mathrm{n}$ & $\%$ \\
\hline ICS & 46 & 76,7 & 2 & 3,3 & 2 & 3,3 & 10 & 16,7 & 60 & 100 \\
\hline ILS & 42 & 70 & 3 & 5 & 4 & 6,7 & 11 & 18,3 & 60 & 100 \\
\hline CS & 41 & 68,3 & 0 & 0 & 3 & 5 & 16 & 26,7 & 60 & 100 \\
\hline ICI & 42 & 70 & 7 & 11,7 & 6 & 10 & 5 & 8,3 & 60 & 100 \\
\hline ILI & 48 & 80 & 1 & 1,7 & 3 & 5 & 8 & 13,3 & 60 & 100 \\
\hline $\mathrm{CI}$ & 60 & 100 & 0 & 0 & 0 & 0 & 0 & 0 & 60 & 100 \\
\hline Total & 279 & 77,5 & 13 & 3,6 & 18 & 5 & 50 & 13,9 & 60 & 100 \\
\hline
\end{tabular}

anteriores ${ }^{3,9,16}$ mostraron que, las dehiscencias y fenestraciones son más frecuentes en las piezas dentarias anteriores que en las posteriores y en las paredes vestibulares, más que en las linguales.

Mediante la evaluación con TCCB, se puso de manifiesto que las dehiscencias se encuentran en mayor proporción en comparación con las fenestraciones en pacientes con maloclusión Clase III, incluso sin necesidad de encontrarse en tratamiento de ortodoncia ${ }^{15,17,18}$, como el estudio de Sun et al. ${ }^{5}$ donde el porcentaje de dehiscencias fue mayor que el de fenestraciones, coincidiendo con el presente estudio. Este resultado se relaciona con el hallazgo encontrado por Kook et al. ${ }^{4}$, donde se observó un menor espesor alveolar a nivel de la UCE en comparación al del ápice en los incisivos inferiores, promoviendo una mayor formación de dehiscencias ${ }^{19}$.

En los pacientes con maloclusión Clase III, la morfología ósea juega un papel importante en la presencia de estos defectos, por presentar paredes óseas más delgadas $\mathrm{e}$ inclinaciones dentarias anteriores que pueden generar la formación de estas alteraciones óseas ${ }^{3,5,9}$. En relación a esto, los estudios de Kook et al. ${ }^{4}$ y Kim et al. ${ }^{20}$, encontraron mayor reabsorción ósea en las corticales óseas mandibulares que en las maxilares de los incisivos de pacientes con maloclusión Clase III con tratamiento de ortodoncia, debido a que la sínfisis mandibular en estos pacientes es más delgada en comparación al resto de maloclusiones. Yagci et al. ${ }^{3}$ también mostraron un porcentaje elevado de dehiscencias mandibulares y el porcentaje de fenestraciones fue mayor en el maxilar. Estos resultados coinciden con los del presente estudio, donde las piezas dentarias con dehiscencias presentaron mayor frecuencia en mandíbula que en maxilar. Y las piezas dentarias con fenestraciones presentaron mayor frecuencia en maxilar que en mandíbula.

En relación a la pieza dentaria más afectada, se observó que, todos los caninos inferiores fueron afectados por dehiscencia, coincidiendo con los estudios de Rupprecht et al. ${ }^{21}$ y Sun et al. ${ }^{5}$. Así también fue observado que la pieza dentaria más afectada por fenestración fue el canino superior, coincidiendo con otros estudios ${ }^{1,5,17}$.
Se sabe que las dehiscencias y fenestraciones presentan una clasificación establecida según la localización que presenten. De esta manera, Yan Yang et al. ${ }^{9}$, clasifica las dehiscencias considerando el tamaño de esta (Clase I), la presencia de otros defectos alveolares (Clase II) o si afecta ambas paredes (labial y lingual/palatino) de la pieza dentaria (Clase III). El grado de severidad se puede manifestar entre las subdivisiones de la Clase I y II, sin embargo, entre las tres clases no se establece cuál puede ser la de peor pronóstico o la más severa. Por otro lado, Pan et al. ${ }^{17}$ clasificó las fenestraciones según el tercio radicular afectado, considerando seis tipos, donde los tres primeros hacen referencia a los tercios apical, medio y coronal de la raíz, los siguientes dos tipos hacen referencia a la exposición de los tercios medio-apical y medio-coronal y el último tipo hace referencia a la exposición de los tres tercios, sin embargo, el tamaño dependerá de la longitud radicular.

El presente estudio, propuso una nueva clasificación de estos defectos, según el tamaño que presentaban, otorgando un grado de severidad para futuros estudios y posibles planes de tratamiento. Se tomaron en cuenta los grados de leve, moderado y severo, debido a que el grado de ausente fue considerado solo como referencia. Entonces, según esta clasificación, las dehiscencias fueron más frecuentes en el grado severo, donde el 100\% de los caninos inferiores evaluados fueron afectados. Las fenestraciones también fueron más frecuentes en el grado severo, donde el 26,7\% de los caninos superiores evaluados fueron afectados. La mayor frecuencia de estos defectos en el grado severo podría darse porque, como menciona Caballero ${ }^{2}$ en su estudio, el movimiento ortodóntico puede agravar esta situación, especialmente el movimiento labio-lingual, debido a la protrusión incisiva superior y retrusión incisiva inferior compensatorias de la clase III. Por ello se debe tener en cuenta que las inclinaciones dentarias que se les realice a los pacientes con maloclusión Clase III esquelética, depende de la gravedad de la maloclusión que presenten, tratando de ubicar el diente lo más equidistante posible de ambas paredes corticales para que el daño periodontal sea el menor posible 22 . 
Por ello, es necesario realizar más investigaciones comparando la presencia de estos defectos óseos antes y después del tratamiento de ortodoncia, así como la edad del paciente, el tipo y la técnica de tratamiento realizado. Esto, para poder evaluar los factores responsables de este aumento en la incidencia. Y así poder realizar un adecuado diagnóstico para un mejor plan de tratamiento, teniendo en cuenta que la existencia de estos defectos puede complicar el tratamiento y producir, incluso, riesgos durante la ortodoncia pre quirúrgica 5 .

Por lo tanto, podemos concluir que los pacientes con maloclusión Clase III con tratamiento de ortodoncia pre-quirúrgica evaluados en el estudio presentaron dehiscencias y fenestraciones vestibulares en las piezas dentarias anteriores superior e inferior. Las dehiscencias estuvieron presentes en todas las tomografías evaluadas, con mayor frecuencia en mandíbula y afectando todos los caninos inferiores en el grado severo. Finalmente, las fenestraciones estuvieron presentes en la mayoría de las tomografías evaluadas, con mayor frecuencia en maxilar $y$ afectando con mayor frecuencia los caninos superiores en el grado severo.

\section{Referencias bibliográficas}

1. Plata E. Repercusiones sobre el hueso alveolar del tratamiento ortodóncico de baja fricción (Damon'). Estudio Clínico Retrospectivo [Tesis de maestría]. [España]: Facultad de Odontología, Universidad Complutense de Madrid; 2013.

2. Caballero S. Dehiscencias y fenestraciones en el tratamiento ortodóncico. Revisión Sistemática. [Trabajo de pre-grado]. [Sevilla]: Facultad de Odontología, Universidad de Sevilla; 2016.

3. Yagci A, Veli I, Uysal T, Ucar FI, Ozer T, Enhos S. Dehiscence and fenestration in skeletal Class I, II, and III malocclusions assessed with cone-beam computed tomography. Angle Orthod. 2012;82(1):67-74.

4. Kook YA, Kim G, Kim Y. Comparison of alveolar bone loss around incisors in normal occlusion samples and surgical skeletal Class III patients. Angle Orthod. 2012;82(4):645-52.

5. Sun L, Zhang L, Shen G, Wang B, Fang B. Accuracy of cone-beam computed tomography in detecting alveolar bone dehiscences and fenestrations. Am J Orthod Dentofac Orthop. 2015;147(3):313-23.

6. Yoshioka T, Kikuchi I, Adorno CG, Suda H. Periapical bone defects of root filled teeth with persistent lesions evaluated by cone-beam computed tomography. Int Endod J. 2011;44(3):245-52.

7. Roque-Torres GD, Meneses-López A, Bóscolo FN, De Almeida SM, Neto FH. La tomografía computarizada cone beam en la ortodoncia, ortopedia facial y funcional. Rev Estomatol Herediana. 2015;25(1):60-77.

8. Proffit WR. Ortodoncia contemporánea. 4ta ed. Barcelona: Elsevier; 2014.
9. Mora MAM, Melgarejo RP, Espinosa GH, Dávila JLS, Chávez JAR. Class III dentofacial alteration treated with camouflage: Clinical case report. Rev Mex Ortodoncia. 2015;3(1):62-9.

10. Lee KM, Kim YI, Park SB, Son WS. Alveolar bone loss around lower incisors during surgical orthodontic treatment in mandibular prognathism. Angle Orthod. 2012;82(4):637-44.

11. Yang Y, Yang $\mathrm{H}$, Pan $\mathrm{H}, \mathrm{Xu} J, \mathrm{Hu}$ T. Evaluation and new classification of alveolar bone dehiscences using conebeam computed tomography in vivo. Int J Morphol. 2015;33(1):361-8.

12. Leung CC, Palomo L, Griffith R, Hans MG. Accuracy and reliability of cone-beam computed tomography for measuring alveolar bone height and detecting bony dehiscences and fenestrations. Am J Orthod Dentofac Orthop. 2010;137(4):S109-19.

13. Asociación Americana de Endodoncia. Glosario de términos endodónticos. 8va ed. México: Asociación Mexicana de Endodoncia [Consultado el 20 de julio 2018]. Accesible en: https://issuu.com/amecee/docs/glosario_ de_terminos_endodont_icos_

14. Wolf H, Edith M, Rateitschak H. Atlas de Periodoncia. 3era ed. Barcelona: Masson; 2005.

15. Hilario K. Frecuencia de dehiscencias y fenestraciones del hueso alveolar según el tipo de maloclusión esquelética en pacientes evaluados con tomografías computarizadas Cone Beam de la Facultad de Odontología de la UNMSM durante el período 2014-2017 [Tesis de Bachiller]. [Perú]: Universidad Nacional Mayor de San Marcos; 2018.

16. Danz JC, Bibby BM, Katsaros C, Stavropoulos A. Effects of facial tooth movement on the periodontium in rats: $A$ comparison between conventional and low force. J Clin Periodontol. 2016;43(3):229-37.

17. Pan HY, Yang H, Zhang R, et al. Use of cone-beam computed tomography to evaluate the prevalence of root fenestration in a Chinese subpopulation. Int Endod J. 2014;47(1):10-9.

18. Marañón G. Límites del tratamiento compensatorio en Ortodoncia. Planteando objetivos reales de tratamiento. Revista Segunda Especialidad Profesional en Ortodoncia y Ortopedia Maxilar. 2014;(2):18-27.

19. Gualan L, Sigüencia V, Bravo M. Maloclusion de clase III, tratamiento ortodóncico. Revisión de la literatura. Rev Latinoam Ortod y Odontopediatria. 2015;(7):1-18.

20. Kim Y, Park JU, Kook YA. Alveolar bone loss around incisors in surgical skeletal class III patients a retrospective 3D CBCT study. Angle Orthod. 2009;79(4):676-82.

21. Rupprecht RD, Horning GM, Nicoll BK, Cohen ME. Prevalence of dehiscences and fenestrations in modern American skulls. J Periodontol. 2001;72(6):722-9.

22. Cardaropoli D, Gaveglio L. The influence of orthodontic movement on periodontal tissues level. Semin Orthod. 2007;13(4):234-45. 